\title{
Protective Mechanical Ventilation, Why Use It?
}

\author{
Emerson Seiberlich, TSA ${ }^{1}$, Jonas Alves Santana ${ }^{2}$, Renata de Andrade Chaves ${ }^{2}$, Raquel Carvalho Seiberlich ${ }^{3}$
}

\begin{abstract}
Summary: Seiberlich E, Santana JA, Chaves RA, Seiberlich RC - Protective Mechanical Ventilation, Why Use It?
Background and objectives: Mechanical ventilation (MV) strategies have been modified over the last decades with a tendency for increasingly lower tidal volumes (VT). However, in patients without acute lung injury (ALI) or acute respiratory distress syndrome (ARDS) the use of high VTs is still very common. Retrospective studies suggest that this practice can be related to mechanical ventilation-associated ALI. The objective of this review is to search for evidence to guide protective MV in patients with healthy lungs and to suggest strategies to properly ventilate lungs with ALI/ARDS.

Contents: A review based on the main articles that focus on the use of strategies of mechanical ventilation was performed.

Conclusions: Consistent studies to determine which would be the best way to ventilate a patient with healthy lungs are lacking. Expert recommendations and current evidence presented in this article indicate that the use of a VT lower than $10 \mathrm{~mL}^{\mathrm{kg}}{ }^{-1}$, associated with positive endexpiratory pressure (PEEP) $\geq 5 \mathrm{cmH}_{2} \mathrm{O}$ without exceeding a pressure plateau of 15 to $20 \mathrm{cmH}_{2} \mathrm{O}$ could minimize alveolar stretching at the end of inspiration and avoid possible inflammation or alveolar collapse.
\end{abstract}

Keywords: Respiration, Artificial; Ventilator-Induced Lung Injury; Pulmonary Atelectasis; Positive Pressure Respiration.

(C2011 Elsevier Editora Ltda. All rights reserved.

\section{INTRODUCTION}

Mechanical ventilation (MV) strategies have been modified over the last decades with a tendency to use increasingly lower tidal volumes (VT) especially in patients with acute lung injury (ALI) or acute respiratory distress syndrome (ARDS). However, in patients without ALI/ARDS the use of high VT is still very common. Retrospective studies suggest that the use of this practice can be related to mechanical ventilation-induced ALI ${ }^{1}$. Due to the lack of consistent prospective studies the ideal management of MV in patients without ALI remains unknown. The objective of this review is to search for scientific evidence to guide protective MV for patients with healthy lungs and to suggest strategies to adequately ventilate lungs with ARDS.
Received from CET/SBA of IPSEMG, Belo Horizonte, Brazil.

1. Anesthesiologist; Instructor of the CET/SBA of IPSEMG; Anesthesiologist of Hospital SOCOR; Anesthesiologist of Hospital das Clínicas da Universidade Federal de Minas Gerais (UFMG); Anesthesiologist of Hospital Vera Cruz Belo Horizonte

2. R3 of the CET/SBA of IPSEMG

3. Respiratory Physiotherapist; Physiotherapist of Hospital Mater Dei Belo Horizonte;

Physiotherapist of CTI Neonatal of Hospital Odilon Behrens de Belo Horizonte

Submitted on August 24, 2010.

Approved on January 31, 2011

Correspondence to:

Dr. Emerson Seiberlich

Rua Bernardo Guimarães 2.138/1.101

Lourdes

30140082 - Belo Horizonte, MG, Brazil

E-mail: seiberlich@gmail.com

\section{ALI AND ARDS}

Acute lung injury was first described in 1967 by Ashbaugh ${ }^{2}$, and it is characterized by refractory hypoxemia, diffuse infiltrates on chest X-ray, and absence of evidence of increased left atrial pressure. Acute respiratory distress syndrome represents the most severe form of ALI. In 1994, the American-European Consensus Conference on $\mathrm{ARDS}^{3}$ defined the criteria for the diagnosis of ALI/ARDS currently used (Box 1).

The etiology of ALI and ARDS varies (Box 2). Mortality ranges from $25 \%$ to $40 \%{ }^{5-7}$, and may reach $58 \%$ in ARDS ${ }^{8}$. The etiology of ARDS influences the prognosis, and sepsis is the condition associated with higher mortality. Other factors that influence mortality include age, degree of organ dysfunction, immunosuppression, chronic liver disease, and severity score (SAPS II - Simplified Acute Physiological Score II) ${ }^{8-10}$, and the higher the number of associated clinical factors, greater the mortality. Furthermore, among patients who survive an episode of ARDS, approximately one third would develop chronic lung disease with restrictive or obstructive pattern ${ }^{11}$.

\section{PATHOPHYSIOLOGY OF ALI/ARDS}

The progression of ALI/ARDS can be divided into two phases. The first, the exudative phase, is associated with diffuse alveolar damage with formation of a protein-rich edema in the alveoli and alveolar interstitium resulting in hypoxemia and reduction of pulmonary complacency.

The alveolar-capillary membrane (ACM) is formed by vascular endothelium and alveolar epithelial cells (type I pneumocytes). It separates the alveolus from the pulmonary capillary blood working as a "barrier" that prevents the leakage 
Box 1 - Definition of ALI and ARDS 4

Acute injury of a suggestive etiology
Severe hypoxemia: $\mathrm{PaO}_{2} / \mathrm{FiO}_{2}<200 \mathrm{mmHg}$ for ARDS, and
$<300 \mathrm{mmHg}$ for $\mathrm{ALI}$
Absence of cardiac failure: wedge pressure $<18 \mathrm{mmHg}$ and CVP
$<4 \mathrm{mmHg}$
Diffuse bilateral infiltrates on chest X-ray

ALI: acute lung injury; ARDS: acute respiratory distress syndrome; $\mathrm{PaO}_{2}$ : arterial oxygen pressure; $\mathrm{FiO}_{2}$ : inspired oxygen fraction; CVP: central venous pressure; PCWP: pulmonary capillary wedge pressure.

Box 2 - Etiology of ALI/ARDS 4

Direct injury factors
Pulmonary infection
Aspiration of gastric contents
Non-fatal drowning
Hyperoxia gas inhalation
Lung contusion
Indirect injury factors
Sepsis/SIRS
Severe non-thoracic trauma
TRALI or massive transfusion
Cardiopulmonary bypass
Pancreatitis
Burns
Shock

ALI: acute lung injury; ARDS: acute respiratory distress syndrome; SIRS: systemic inflammatory response syndrome; TRALI: transfusion-related acute lung injury.

of intravascular fluid to the alveolar space. During the exudative phase the breakdown in intercellular junctions compromises the "barrier" function ${ }^{12}$, resulting in deposition of fibrin and formation of intra-alveolar hyaline membrane.

The magnitude of the alveolar damage in ARDS results from an imbalance between the pro-inflammatory and antiinflammatory responses in face of the initial injury. Both direct (pulmonary) and indirect (extra-pulmonary) aggressions induce the release of humoral and cellular inflammatory mediators. Activation of monocytes and macrophages by primary cytokines, tumor necrosis factor $\alpha$ (TNF- $\alpha$ ) and interleukin (IL) 1- $\beta$ culminates with the release of secondary cytokines and other mediators that lead to a systemic inflammatory response and the release of proteolytic and oxidizing enzymes. The final result is the dysfunction and death of alveolar epithelial cells.

The second phase of ALI/ARDS is known as fibroproliferative, and it is associated with fast proliferation of type II pneumocytes and fibroblasts. The actions of fibroblasts result in deposition of collagen and proteoglycans in the hyaline membrane reducing pulmonary complacency and increasing the pathological dead space. The pulmonary capillary bed can be obstructed leading to pulmonary hypertension and dysfunction of right cardiac chambers.

\section{PROTECTIVE MV IN ALI AND ARDS}

In a pioneer study comparing mechanical ventilation strategies in patients with ALI/ARDS, Ranieri et al. ${ }^{13,14}$ found that the use of smaller tidal volumes reduces the concentration of inflammatory mediator both in bronchoalveolar washing fluid and systemic circulation. Other studies confirmed that this practice would alter the final outcome of these patients ${ }^{15-17}$. In the main study, the ARDs Network ${ }^{18}$, the use of low (6 mL. $\mathrm{kg}^{-1}$ of predictive body weight) and high (12 mL. $\mathrm{kg}^{-1}$ of predictive body weight) tidal volumes were compared. The use of low VT with a maximum plateau pressure of $30 \mathrm{cmH}_{2} \mathrm{O}$ resulted in a lower intra-hospital mortality (31\% versus $39 \%$ ) and a lower number of days on mechanical ventilation. The benefit in patient survival remained after a 6-month follow-up.

A new study of the ARDS Network ${ }^{19}$ undertaken four years later assessed the use of high positive end-expiratory pressure (PEEP) $\left(13.2 \pm 3.5 \mathrm{cmH}_{2} \mathrm{O}\right)$ versus low PEEP $\left(8.3 \pm 3.2 \mathrm{cmH}_{2} \mathrm{O}\right)$ in patients with ARDS on protective ventilation ( $6 \mathrm{~mL} . \mathrm{kg}^{-1}$ of predictive weight and plateau pressure $\leq 30 \mathrm{cmH}_{2} \mathrm{O}$ ). A significant statistical difference in mortality, number of days on mechanical ventilation, or degree of organ dysfunction was not observed between groups.

During the use of protective ventilation in ARDS the development of hypercapnia and respiratory acidosis may be expected as part of this approach. This change when foreseen is called permissive hypercapnia. In an attempt to compensate for these changes one can try using more elevated respiratory rates. The fall in $\mathrm{pH}$ up to 7.15 is usually well tolerated with none or small changes in cardiac output and blood pressure 5,20 . Situations in which permissive hypercapnia may be harmful include intracranial hypertension, severe concomitant metabolic acidosis, severe pulmonary hypertension, right ventricular failure, and coronary syndromes.

The use of mechanical ventilation in the prone position in ARDS seems to improve oxygenation and alveolar recruitment, but benefits on mortality have not been observed ${ }^{21}$. The prone position improves the ventilation/perfusion ratio if the most compromised alveolar units are in the dependent position. Note that ventilation in this position leads to inadvertent extubation and loss of venous accesses.

Recommendations for protective ventilation in patients with ARDS are summarized in Box 3.

\section{OXYGEN TOXICITY}

Human and animal studies suggest that the administration of supplementary oxygen $\left(\mathrm{O}_{2}\right)$ may lead to different aspects of airways injuries. The effects of hyperoxia in the lungs have been known for some time. It has been demonstrated that it causes the formation of alveolar hyaline membrane, edema, hyperplasia, proliferation of type II pneumocytes, destruction 
Box 3 - Guidelines for protective ventilation in patients with ALI/ARDS 22

Pressure-controlled or volume-controlled mode demonstrated the
same efficacy in this population. The experience of the physician
and the correct interpretation of physiologic changes are the most
important elements when choosing the ventilation mode.
Reduction of the tidal volume until approximately 6 mL.kg-1
of predictive body weight; maintaining a pressure plateau
$\leq 30 \mathrm{cmH}_{2} \mathrm{O}$ reduces mortality and it is strongly recommended
(ARDS NETWORK 2000).
Increasing PEEP in an attempt to reduce FiO ${ }_{2}$ is not supported by
current studies. Animal studies suggest the use of high PEEP
to avoid pulmonary derecruitment, but how to define the optimal
values for this purpose has not been elucidated. Recruitment
maneuvers seem to be a possibility for patients who respond to
elevated PEEP levels, but they are not indicated in all patients.
Careful hemodynamic monitoring should be maintained in patients
in protective mechanical ventilation due to the risk of compromis-
ing tissue perfusion by adjusting those parameters.
Hypercapnia due to protective mechanical ventilation (permissive
hypercapnia) is usually well tolerated and it is more desirable than
the use of elevated tidal volumes or elevated plateau pressures.
Contraindications to permissive hypercapnia include intracranial
hypertension, concomitant severe metabolic acidosis, worsening
pulmonary hypertension, right ventricular failure, and acute coro-
nary syndromes 4 .

ALI: acute lung injury; ARDS: acute respiratory distress syndrome; PEEP: positive end-expiratory pressure; $\mathrm{FiO}_{2}$ : inspired oxygen fraction.

of type I pneumocytes, interstitial fibrosis, and pulmonary vascular remodeling. The formation of reactive oxygen species in mitochondria is regarded as the main cause of the diffuse alveolar damage seen in animals exposed to high fractions of inspired oxygen ${ }^{21}$. In studies of humans with lung diseases it is difficult to define the role of this toxicity when facing so many variables. However, in the study of the ARDS Network mentioned above ${ }^{19}$, which compared high PEEP versus low PEEP, the 273 patients with ARDs ventilated with low PEEP required higher $\mathrm{FiO}_{2}$ when compared to those ventilated with high PEEP $(n=276)$ according to the protocol proposed by the investigators. However, a statistically significant difference in mortality before and after discharge from the hospital and secondary outcomes was not observed. Note that that study was not designed to evaluate the outcome as a function of the $\mathrm{FiO}_{2}$ values but of PEEP values.

\section{MECHANICAL VENTILATION-RELATED ALI/ARDS}

Previously, lung damage attributed to elevated VTs was described as a possibility of air extravasation into the pleural space. When extravasation occurs due to very elevated pressure in the airways it characterizes barotrauma. More recently, other forms of MV-associated damages have been described. Volutrauma results from alveolar hyperdistension leading to a local inflammatory process. Atelectrauma is a consequence of the alveolar injury caused by stress on the ACM when facing an instable recruitment/derecruitment at each ventilatory cycle. Biotrauma is caused by the local and systemic inflam- matory responses resulting from aggression caused by both volutrauma and atelectrauma or the combination of both ${ }^{24}$.

Animal studies revealed that the use of high VTs in healthy lungs leads rapidly to pulmonary changes similar to those seen in ARDS. The injury cause by MV results in alveolar damage with consequent edema of the alveolar-capillary membrane, release of inflammatory mediators in the systemic circulation, and activation and dislocation of inflammatory cells into the alveoli ${ }^{1}$.

The deleterious effects of high VTs have been observed even in patients ventilated for a short time. Fernadez et al. ${ }^{25}$ collected the intraoperative VTs of patients undergoing pneumonectomies. According to them, $18 \%$ of patients developed postoperative acute respiratory failure (ARF), and in half of these cases patients received the diagnosis of ALI/ARDS. After analyzing the data they observed that patients who were ventilated with higher VTs (mean of $8.3 \times 6.7 \mathrm{~mL}^{-\mathrm{kg}^{-1}}$ of ideal body weight, $p<0.0001$ ) developed ARF. Logistic regression analysis identified the use of high intraoperative VTs and higher intravascular fluid replacement as risk factors for postoperative ARF.

On a study of 52 patients, Mechelet et al. ${ }^{26}$ compared interleukins levels, IL-1, IL-6, and IL-8 in patients undergoing esophagectomies to treat cancer, ventilated with conventional MV (VT 9 mL. $\mathrm{kg}^{-1}$ of ideal body weight without PEEP) and protective MV (VT $5 \mathrm{~mL} . \mathrm{kg}^{-1}$ of ideal body weight and PEEP of $5 \mathrm{cmH}_{2} \mathrm{O}$ ). Patients who were on protective MV had lower levels of inflammatory factors both at the end of monopulmonary ventilation and 18 hours after surgery. Protective MV also resulted in better $\mathrm{PaO}_{2} / \mathrm{FiO}_{2}$ ratio during monopulmonary ventilation and 1 hour after surgery in addition a reduction in postoperative MV time.

A randomized clinical assay ${ }^{27}$ with surgical patients admitted to the ICU compared VT of 12 and $6 \mathrm{~mL}^{\mathrm{kg}} \mathrm{kg}^{-1}$ of ideal body weight. Patients on the postoperative period of neurosurgery and cardiac surgery were excluded. Patients ventilated with lower VTs had fewer infections, less time of MV, and a shorter stay on the ICU.

The Third Brazilian Consensus on Mechanical Ventilation ${ }^{28}$, published in 2007, mentions intraoperative mechanical ventilation in patients without lung disease, and recommends the use of PEEP $\geq 5 \mathrm{cmH}_{2} \mathrm{O}$ during general anesthesia (degree of recommendation $\mathrm{B}$ ), alveolar recruitment maneuvers (degree of recommendation $\mathrm{B}$ ), $\mathrm{FiO}_{2}$ between $30 \%$ and $40 \%$ or the lower $\mathrm{FiO}_{2}$ to maintain oxygen saturation above $98 \%$ (degree of recommendation $\mathrm{C}$ ), and not using high tidal volumes ${ }^{28}$.

A recent study by Soubhie et al. ${ }^{29}$, published on the Brazilian Journal of Anesthesiology, evaluated transoperative ventilatory modalities used by anesthesiologists in Brazil through a questionnaire. They demonstrated that $94 \%$ routinely use PEEP while $86.5 \%$ use $\mathrm{FiO}_{2}$ between $40 \%$ and $60 \%$. Intraoperative alveolar recruitment maneuvers were performed by $78.4 \%$, but only $30 \%$ used protective mechanical ventilation with VT lower than $7 \mathrm{~mL} . \mathrm{kg}^{-1}$.

It should be emphasized that the expression "Iow VT" should in reality be "normal VT" since mammals usually have 
a VT of approximately $6.3 \mathrm{~mL} . \mathrm{kg}^{-1}$. In most studies tidal volume is calculated based on the predictive body weight whose variables are the gender and height of the patient. This is very important to avoid over- or underestimating the calculated VT for MV (Box 4) ${ }^{1}$.

Box 4 - Calculation of predictive body weight in kilograms $(\mathrm{kg})^{1}$ \begin{tabular}{|l|l}
\hline Male gender & $50+0.91 \times$ (height in centimeters -152.4$)$
\end{tabular} Female gender $\quad 45.5+0.91 \times$ (height in centimeters -152.4 )

\section{CONCLUSION}

Consistent studies to determine which would be the ideal ventilation mode for a patient with healthy lungs are lacking. Expert recommendations and current evidence presented here indicate the use of a VT lower than $10 \mathrm{~mL} \cdot \mathrm{kg}^{-1}$ of ideal body weight associated with PEEP $\geq 5 \mathrm{cmH}_{2} \mathrm{O}$, without exceeding a plateau of 15 to $20 \mathrm{cmH}_{2} \mathrm{O}$, would minimize end-inspiratory alveolar stretching and avoid possible inflammation or alveolar collapse.

It is important to emphasize that in some patients with healthy lungs exposed to MV for a short period for low risk procedures a VT of $10 \mathrm{~mL} . \mathrm{kg}^{-1}$ may not cause end-inspiratory alveolar stretching, and therefore it does not have the consequences mentioned. On the contrary, when these patients are ventilated with a pressure plateau $<15 \mathrm{cmH}_{2} \mathrm{O}$ without PEEP the use of low VTs can lead to atelectasis. A high enough PEEP should be used in these cases to avoid this occurrence and possible oxygenation compromise. The same does not happen to patients breathing spontaneously. In this case even with low pressure plateau transalveolar pressure maintains the "negative" pleural pressure avoiding alveolar collapse. 